\title{
Improving Pediatric Hearing Testing for Children with Developmental Delays: The Effects of Video Modeling on Child Compliance and Caregiver Stress
}

\author{
Megan Y. Roberts ${ }^{1}$ Jennifer Rosenwasser ${ }^{1} \quad$ Jennifer Phelan ${ }^{1} \quad$ Lauren H. Hampton ${ }^{1}$ \\ ${ }^{1}$ The Roxelyn and Richard Pepper Department of Communication \\ Sciences and Disorders, Northwestern University, Evanston, IL \\ Address for correspondence Megan Y. Roberts, Department of \\ Communication Sciences and Disorders, Evanston, IL 60208 \\ (e-mail: megan.y.roberts@northwestern.edu). \\ J Am Acad Audiol 2020;31:310-316.
}

\begin{abstract}
Keywords

- pediatric audiology
\end{abstract}

\section{Introduction}

The age at which a child is diagnosed with a hearing loss can greatly impact speech and language development (Moeller) ${ }^{20}$. For children with developmental delays (DDs) of any kind, it is essential to understand if a hearing loss may be contributing to these delays. DDs may be due to an autism spectrum disorder (ASD), Down syndrome, a hearing loss, or unknown causes or a combination of etiologies. Children with DD may have difficulty understanding directions, may be uncomfortable with a new situation or may simply not want to complete the hearing testing tasks. Regardless of the reasons, noncompliant behaviors have a negative impact on hearing testing, which requires reliable behavioral responses from the child. The purpose of this study was to test a video modeling intervention to improve hearing testing compliance in toddlers with DD.
Copyright $\odot 2020$ by the American Academy of Audiology. All rights reserved. Thieme Medical Publishers, Inc., 333 Seventh Avenue, New York, NY 10001, USA. Tel: +1(212) 760-0888.
DOI https://doi.org/ 10.3766/jaaa. 18070 ISSN 1050-0545. 


\section{The Importance of Hearing Testing for Children with DD}

Hearing loss may be more prevalent in children with DDs $(\text { Kwok et al })^{18}$. As many as $8 \%$ of children with ASD experience hearing loss (Fitzpatrick et al) ${ }^{13}$, which may be due to an increased prevalence of serous otitis media (Rosenhall et al) ${ }^{23}$. ASD, DD, and hearing loss present similar characteristics with regards to language delays and impaired social communication (e.g., failing to respond to own name). These similarities may be a contributing factor to delayed diagnoses of hearing loss in children with ASD (Fitzpatrick et al) ${ }^{13}$. As such, all children with DD should receive a comprehensive pediatric hearing evaluation to determine if a hearing loss may be contributing to the delay. This comprehensive pediatric hearing evaluation includes a test battery of speech detection and recognition measures, pure-tone air conduction audiometry across the frequency range, tympanometry, and otoacoustic emissions (Joint Committee on Infant Hearing) ${ }^{16}$. These tests are categorized as either behavioral or objective. Behavioral tests require a child to respond to the sounds that they hear, whereas objective measures require no behavioral response, but rather a physiologic response from the child. Both types of tests are used in pediatric hearing evaluations to obtain a complete understanding of the child's hearing sensitivity. Although behavioral audiometry requires a child's response to different speech and frequency stimuli, objective measures also require the child to comply with directions (i.e., sitting still while the audiologist inserts a testing instrument into the ear). In addition, objective measures, such as otoacoustic emissions, do not provide an estimate of hearing thresholds.

\section{Hearing Testing Challenges for Children with DD}

Children with DD present with unique challenges for audiologists, which may make obtaining a valid hearing assessment difficult For example, many children with DD present with language and cognitive differences, which hinders their ability to understand the directions of hearing tasks thereby prohibiting the use of threshold measurement procedures (Downs et al) $^{11}$. In addition, some children with DD may exhibit trouble attending for a period long enough to obtain valid hearing testing results (Fitzpatrick et al) ${ }^{13}$. Furthermore, visual reinforcement audiometry requires the child to respond to the reinforcer in a consistent manner across a sufficient number of trials to obtain a conditioned response, which is less likely for children with DD who have reduced attention spans. In addition, some children with DD habituate to stimuli, which may give more false-positive and falsenegative responses, leading to poor reliability of the test results (Demopoulos and Lewine) ${ }^{10}$. If the child is not responsive to the conditioning of the hearing tasks, then behavioral test measures cannot be obtained reliably. Many children with DD, especially children with ASD, also have difficulty with change and become fearful in new environments (Downs et al) ${ }^{11}$. For example, the enclosed nature of a sound booth may cause the child to become fearful and withdrawn or even agitated. Furthermore, many children with ASD display sensory difficulties, such as hyperacusis, which is an increased sensitivity to sounds (Rosenhall et al) 23. If a child does not tolerate their ears being touched or wearing headphones, there is no way to obtain ear-specific, behavioral, or objective test measures. Finally, children with DD often exhibit disruptive behaviors (e.g., hitting, screaming, and biting) that may make it difficult to obtain accurate audiometric data (Baker et al) ${ }^{3}$.

When these difficulties prevent an accurate evaluation of a child's hearing abilities, auditory brainstem response (ABR) is often recommended because no behavioral response is required (Davis and Stiegler) ${ }^{9}$. However, ABRs require general anesthesia, which is not optimal for children already experiencing DD, given that repeated exposures to anesthesia is associated with higher rates of learning disabilities (Padish-Clarin and Hawkins) $^{21}$. In addition, ABR threshold measurements may underestimate the severity of hearing loss when the hearing loss is greater than a moderate loss and may overestimate hearing loss in people with normal hearing (Demopoulos and Lewine $)^{10}$. Given the limitations of $A B R$ testing, it is important to consider ways in which to support pediatric behavioral hearing testing.

\section{Video Modeling}

Given these increased complexities of assessing children with DD, it is important to identify strategies that maximize successful completion of hearing testing for children with DD. One potential solution is the use of video models, as children with DD often learn better visually than auditorily (Demopoulos and Lewine $)^{10}$. Through video modeling, an action or interaction is modeled, video recorded, and then viewed. Video modeling has been found to be effective for improving a variety of skills (e.g., social communication and self-help) for individuals with DD (Bellini and Akullian) ${ }^{5}$, but to our knowledge, it has not been used with hearing testing. Increased exposure to a new activity is associated with increased compliance in children with DD (Charlop-Christy et al $)^{7}$. As such, children with DD may benefit from multiple exposures to the hearing testing setting and tasks by watching video models. This increased familiarity may increase testing compliance, which in turn may reduce the number of referrals for sedated ABRs.

Video modeling may also reduce parental stress associated with the hearing evaluation. This is particularly important for parents of children with DD, who experience more stress than parents of typically developing children (Baker et al) ${ }^{3}$. Increased stress may be due to increased financial needs, isolation stemming from difficulties interacting with other children, reduced adaptive skills, and increased disruptive behaviors (Baker et al) ${ }^{4}$. By exposing the parents to hearing tasks and test-ing environment, they may feel more prepared to support their child's compliance during the hearing tasks. For children with ASD, interventions that directly target a reduction in caregiver stress facilitate improvement in the child's ASD related behaviors (Kayfitz et al) ${ }^{17}$.

\section{Purpose of This Study}

To date, there is little information about effective strategies to improve compliance during hearing evaluations of children 
with DD. Thus, the purpose of the current study was to determine if pre-exposure to hearing testing through video modeling would enhance testing compliance in children with DD. A secondary aim of the study was to examine the impact of video modeling on caregiver stress. The following research questions guided the present study: Does video modeling for children with DD before hearing testing (a) increase the compliance with the assessment and (b) decrease caregiver stress regarding the evaluation?

\section{Method}

\section{Design}

The current study was a double-blind randomized clinical trial (NCT03002363). The study was conducted at the Northwestern University Center for Audiology, Speech, Language, and Learning (NUCASLL) in Evanston, IL. The trial was approved by the Northwestern University Institutional Review Board (STU00203250). All caregivers provided written informed consent to par-ticipate in the study.

\section{Participants}

Participants included 24 children and their caregivers. Participants were recruited continuously between June 2016 and June 2017 from the NUCASLL Developmental Diagnostic Program, a medical diagnostic evaluation team through Illinois' Early Intervention Program. All children were referred to the Developmental Diagnostic Program by their service coordinators based on: (a) significant and unexplained DDs, (b) lack of progress, (c) unexpected regression, or (d) atypical development that could not be explained based on known medical, developmental etiology. A hearing evaluation is required as part of the medical diagnostic process for children without a comprehensive hearing evaluation within the last 2 years. Eligibility criteria included (a) no previous comprehensive hearing evaluation, (b) chronological age of 36 months or less, and (c) English as the primary language spoken at home. At the time of inclusion, diagnosis was still unknown. Twenty-seven caregivers were contacted to participate in the study; 24 (89\%) agreed to participate. Participant characteristics are provided in -Table 1.

The full developmental evaluation was completed by a multidisciplinary team of expert clinicians comprising a developmental and behavioral pediatrician, a developmental therapist, and a speech-language pathologist following the audiology examination. The diagnosis was based on a developmental evaluation, language assessment, and the DSM-5 diagnostic criteria (American Psychiatric Association) ${ }^{2}$. Children with an ASD DD presented with impairments in social communication (i.e., maintaining reciprocal social interactions) and restricted or repetitive behaviors (i.e., atypical sensory interests or sensitivities). Children with a non-ASD DD presented with delays in several developmental domains (i.e., cognition and language) but did not meet the diagnostic criteria for ASD. When making the diagnosis, clinicians considered caregiver reports during semistructured interviewing, developmental and medical histories, observational impressions of the child, and performance during the Autism Diagnostic Observation Schedule-Second Edition (Autism Diagnostic Observation Scale-2; Lord et al) ${ }^{19}$.

\section{Randomization}

Eligible participants were stratified in a 1:1 randomization scheme to intervention and control arms by using a computergenerated random number sequence. The randomization

Table 1 Participant Characteristics

\begin{tabular}{|c|c|c|c|}
\hline \multirow[t]{2}{*}{ Variable M (SD) [Range] } & \multicolumn{2}{|l|}{ Trial Arm } & \multirow{2}{*}{$\begin{array}{l}\text { Difference } p \text {-Value } \\
\text { (Effect Size) }^{*}\end{array}$} \\
\hline & Intervention $(\mathrm{n}<12)$ & Control $(n<12)$ & \\
\hline Females, $n$ & 5 & 0 & \\
\hline Age, months & $30.4(3.7)[24-35]$ & $30.6(4.1)[23-35]$ & $0.750(0.05)$ \\
\hline \multicolumn{4}{|l|}{ Race, N } \\
\hline Asian & 4 & 3 & \\
\hline Black & 0 & 2 & \\
\hline White & 8 & 6 & \\
\hline Other & 40 & 1 & \\
\hline Diagnosis of $\mathrm{ASD}^{\dagger} \mathrm{n}$ & 10 & 9 & \\
\hline Diagnosis of non-ASD DD ${ }^{\dagger} \mathrm{n}$ & 2 & 3 & \\
\hline $\begin{array}{l}\text { Autism Diagnostic Observation } \\
\text { Scale algorithm score }{ }^{\dagger}\end{array}$ & $19.2(5.1)[11-28]$ & $20.7(3.6)[14-27]$ & $0.460(0.34)$ \\
\hline $\begin{array}{l}\text { Preschool Language Scale } \\
\text { (Zimmerman et al, 2011) standard score! }\end{array}$ & $64.75(13.38)[50-100]$ & $60.18(5.90)[52-72]$ & $0.250(0.44)$ \\
\hline No. of times watched video & $2(1.38)[1-5]$ & $1.75(1.01)[1-4]$ & $0.530(0.21)$ \\
\hline $\begin{array}{l}\text { No. of days between watching } \\
\text { video and hearing testing }\end{array}$ & $3.2(3.7)[0-13]$ & $4.6(4.1)[1-13]$ & $0.390(0.38)$ \\
\hline
\end{tabular}

*Effect size $=$ Cohen's d.

${ }^{\dagger}$ Diagnosis made/assessment given within 6 weeks after the audiology examination. 
sequence was unknown to the audiologist and the study coordinator who enrolled participants and assigned participants to experimental groups. After the study coordinator obtained verbal consent, random assignment occurred using Research Electronic Data Capture (Harris et al) ${ }^{15}$.

\section{Videos}

Once randomly assigned, 2 weeks before the hearing evaluation, caregivers received an email containing instructions and a link to watch a 2.5-minute video. Caregivers also received a phone call reminder 3 days before the appointment. Caregivers were instructed to watch the video with their child at least one time entirely, between the time that consent was obtained and the day of their hearing evaluation. In addition, participants were able to watch the video multiple times. Parents reported the timing and frequency of video viewing, which is provided in - Table 1.

The control video included information on human and animal ears, hearing, and the negative effects of loud noise on hearing and overall hearing health, but nothing related to the evaluation. The control video is available at https:// youtu.be/Un7E8e5391A. The intervention video model was designed to promote pre-exposure to all aspects of the hearing evaluation in a social story format (Gray et al) ${ }^{14}$. The intervention video also included strategies for the caregiver such as practice with headphones and desensitizing the child's ears through touch. The intervention video is available at https://youtu.be/776ep_LzAc0. The control and intervention videos were identical in length and all aspects, except for the content. On average, participants in both groups watched the video the same number of times (twice) and watched the video at similar time before the hearing evaluation (3-4 days before the hearing evaluation) (see - Table 1).

\section{Measures}

The primary outcome measure of this study was the total assessment completion score. A total number of five tests were attempted during each visit. - Table $\mathbf{2}$ provides a summary of the measures, and how the overall assessment completion score was calculated for each participant. Scores ranged from 0 (no assessments were completed) to 16 (all tasks for all assessments were completed). The same audiologist, naive to the experimental condition, performed the hearing evaluation for every participant. All aspects of the hearing testing occurred at NUCASLL in a sound-treated test suite.

The evaluation used a two-tester model, with a boardcertified pediatric audiologist administering each test and an audiology graduate student as an assistant. The pediatric audiologist has more than 10 years of experience testing young children, including those with DDs. She also completed a Leadership in Education in Neurodeve- lopmental and Related Disabilities Fellowship, which is an interdisciplinary training to improve the health of children with disabilities. The same audiology graduate student was present for all assessments. During the appointment, the child sat on the caregiver's lap while the audiologist performed each test. Otoscopy was conducted before placing anything in the child's ear canal. An Otometrics MADSEN Astera2 audiometer (Natus Medical Inc., Schaumburg, IL) was used for all behavioral assessments. Etymotic Research ER-14B 10-mm disposable foam ear tips (Etymotic Research, Inc., Elk Grove, IL) or TDH-39 phones (Telephonics Corporate Headquarters, Farmingdale, NY) were used when possible. If the child did not tolerate the ear tips, sound field speakers were used. Only three children tolerated ear tips (two in the video modeling group and one in the control group). The behavioral assessment consisted of a Speech Awareness Threshold (SAT) and visual reinforcement audiometry with a mechanical toy reinforcer. Reliability of behavioral responses was based on the experienced audiologist's confidence that the child's response was not by chance. Acquisition of sound field thresholds or ear-specific thresholds at $500 \mathrm{~Hz}, 1000 \mathrm{~Hz}$, and $4000 \mathrm{~Hz}$ were attempted on every child (Tharpe et al) ${ }^{24}$. Warble tones were used when presenting tonal stimuli in the sound field to avoid the problem of standing waves. An Interacoustics Titan was used for all objective test measures. Tympanometry was conducted using a $226-\mathrm{Hz}$ probe tone and distortion pro duct otoacoustic emissions (DPOAEs) were conducted from 1500 to $8000 \mathrm{~Hz}$ to measure the outer hair cell cochlear function. The order in which the tests were conducted was flexible to accommodate

Table 2 Assessment Completion

\begin{tabular}{|l|l|l|l|l|}
\hline Measure & Criterion & Scoring & $\begin{array}{l}\text { Points } \\
\text { Possible }\end{array}$ \\
\cline { 3 - 5 } & & Insert Ear Phones & Sound Field & \\
\hline Otoscopy & Visualization of the ear canal and tympanic membrane & 1 point per ear & 2 \\
\hline SAT & Two reliable responses at the child's threshold & 1 point per ear & $\begin{array}{l}2 \text { points if } \\
\text { obtained }\end{array}$ & 2 \\
\hline $\begin{array}{l}\text { Pure-tone } \\
\text { air conduction }\end{array}$ & $\begin{array}{l}\text { Two reliable responses for each frequency } \\
\text { tested, at the child's threshold }\end{array}$ & $\begin{array}{l}1 \text { point per } \\
\text { frequency per ear }\end{array}$ & $\begin{array}{l}2 \text { points per } \\
\text { frequency }\end{array}$ & 8 \\
\hline Tympanometry & A reliable tympanogram & $\begin{array}{l}1 \text { point per } \\
\text { ear obtained }\end{array}$ & 2 \\
\hline $\begin{array}{l}\text { DPOAEs } \\
\text { Total possible } \\
\begin{array}{l}\text { assessment } \\
\text { completion score }\end{array}\end{array}$ & A complete run of DPOAEs from $1.5 \mathrm{kHz}$ to $8 \mathrm{kHz}$ & $\begin{array}{l}1 \text { point per } \\
\text { ear obtained }\end{array}$ & 2 \\
\hline
\end{tabular}


differences and preferences between participants (Tharpe et al $)^{24}$. The decision about the test order, which was individualized based on child preferences and performance, was applied systematically across all children. Given that the audiologist was the same between the two experimental groups and that participants were randomly assigned to groups, the order of tests did not vary between experimental groups.

Caregiver stress about the child's hearing evaluation was measured immediately before and immediately after the hearing evaluation. Before the hearing evaluation, parents were asked to rate the following statement on a 5-point Likert scale: Please describe your stress level regarding your child's upcoming hearing testing. After the hearing evaluation, the parents were asked to rate this following statement in the same 5-point scale: Please describe your stress level during your child's hearing testing. The 5-points were anchored as follows:

(a) no stress at all, (b) a little stressed, (c) somewhat stressed, (d) much stressed, and (e) very much stressed.

\section{Statistical Analyses}

All data were collected on paper and entered into Research Electronic Data Capture (Harris et al) $)^{15}$. A linear regression controlling for pretest stress scores was performed for all outcomes with the experimental group as the independent variable and hearing assessment completion and caregiver stress as the dependent variables. All data analyses were completed using RStudio version 3.3.3 and an alpha set at 0.05 (R Core Team) $)^{22}$.

\section{Results}

- Table 1 summarizes the participant characteristics. Differences in group characteristics revealed an unequal distribution of females, all randomly assigned to the intervention arm; no other differences between groups were observed. Eighty percent of participants in each group were diagnosed with ASD; the remaining $20 \%$ were diagnosed with a DD.

Video modeling did not significantly increase hearing assessment completion $(t=1.4, p=0.17, d=0.68)$. On average, children in the intervention group received an assessment completion score of 11 out of 16 (69\%), whereas children in the control group received, on average, a score of 8.5 out of 16 (53\%). This difference corresponds to an effect size of $d=0.68$. These results are presented in - Table 3 . We also explored the extent to which compliance varied by the type of test (objective or subjective). However, there was no difference in hearing assessment completion scores by test type $(t=2.1, p=0.18)$.

As seen in - Table 3, caregivers in the intervention group reported significantly less stress on the 5-point scale (1.58) after the appointment as compared with caregivers in the control group (2.67), and did not report different levels of anticipated stress before the appointment (2.08 and 2.17 at pretest). When controlling for pretest stress ratings, between-group differences for the reported caregiver stress level remained significantly different $(t=-2.754 ; p<0.05)$.

\section{Discussion}

This was the first study to empirically test an intervention to improve hearing testing compliance in children with DD. The comparison of hearing assessment completion scores between the treatment and control groups suggest that results failed to reach statistical significance in the small sample of participants. These results align with findings from other studies related to compliance in children with ASD. For example, video modeling alone did not increase completion of test items on a gross motor assessment in children with autism (Case and Yun) 6 . However, when video modeling was combined with other behavioral strategies such as a preference assessment, children with ASD were compliant with dental procedures (Cuvo et al) ${ }^{8}$. Taken together, these findings suggest that children with DD may benefit from a multicomponent intervention that includes video modeling as one of several components.

Importantly, caregiver stress was significantly reduced for caregivers in the treatment group, which may indicate that the video model may have improved the overall quality of and satisfaction with hearing testing, which was not otherwise measured. These results are similar to prior work on video modeling to reduce anxiety related to health procedures. For example, older typical children (8-16 years) who watched a video recording of a child using a nasal mask for inhalation sedation for a dental treatment had significantly less anxiety than those children who watch an oral hygiene video (Al-Namankany et al) ${ }^{1}$.

Table 3 Outcome Comparison: Treatment vs. Control

\begin{tabular}{|l|l|l|l|l|l|l|}
\hline \multirow{2}{*}{ Outcome } & \multicolumn{2}{|l|}{ Mean (SD) } & Difference & $\boldsymbol{t}$ & $\boldsymbol{p}$ & \multirow{2}{*}{ Effect Size (d) } \\
\cline { 2 - 5 } & Treatment (12) & Control (12) & & & & \\
\hline Otoscopy & $1.8(1.0)$ & $0.6(0.9)$ & 0.4 & 1.1 & 0.31 & 0.43 \\
\hline SAT & $2(1.3)$ & $1.6(1.0)$ & 0.5 & 1.5 & 0.14 & 1.07 \\
\hline Pure-Tone air conduction & $5.4(3.6)$ & $3.8(3.9)$ & 1.6 & 1.0 & 0.31 & 0.44 \\
\hline Tympanometry & $1(0.8)$ & $1.75(0.6)$ & -0.1 & -0.3 & 0.77 & -0.12 \\
\hline DPOAEs & $1.4(0.9)$ & $1.0(1.0)$ & 1.4 & 1.1 & 0.30 & 0.43 \\
\hline Assessment completion & $11.3(2.1)$ & $8.5(5.4)$ & 2.8 & 1.4 & 0.17 & 0.68 \\
\hline Caregiver stress & $1.6(0.8)$ & $2.7(1.4)$ & 1.1 & -2.4 & 0.03 & 0.97 \\
\hline
\end{tabular}


Strengths of this study include (a) the consistent clinical care between all participants throughout the study, (b) masking of experimental condition, (c) a standardized research protocol across experimental conditions, (d) a community-based sample with high rates of participation, and (e) a sample of toddlers with a range of cognitive abilities. However, the current findings should be considered within the context of several limitations. Whereas we collected data as to the number of times the video was viewed, we did not collect data related to the children's attention to the video. Given the limited attention span of children with $\mathrm{DD}$, it is possible that the video modeling strategy should focus on parents rather than the child. For example, if a parent knows what to expect during the visit, they may be better prepared to support their child during the visit. Furthermore, because of the many components of the video model (exposure to the audiologist, exposure to the testing environment, exposure to the testing instruments, and caregiver strategies), the active ingredients of the video model strategy remain unknown. It is unknown if parents used the de-sensitization strategies recommended in the video. It is possible that the extent to which the parents used these strategies with their toddler, the number of times the video was viewed, or the proximity of viewing relative to the hearing evaluation may have affected the results. However, because of the limited sample size, the current study did not examine the extent to which the number of times the parent viewed the video impacted outcomes. In addition, the small sample size may have resulted in a type II error (failing to reject the null hypothesis, when the null hypothesis is false). There was also an unequal distribution of gender among the intervention and control groups, which may have impacted the results. Because girls with autism often exhibit different behavioral characteristics (Dworzynski et al) ${ }^{12}$, it is possible that video modeling may be more effective for children with specific characteristics (e.g., children with higher attentional skills). Finally, a more comprehensive measure of caregiver stress may better assess the impact of video modeling on caregiver stress during audiology examinations.

Future research should expand the current study in three important ways. First, future research should give parents more specific guidelines about the number of times to watch the video, should use software to control the number of participant viewings, and should include a measure of the child's attention to the video model. Second, a measure of child and parent stress that provides more specificity and variability to detect the subtle changes in stress over the course of the study could be useful. Third, the use of the video modeling procedures for specific populations of children with DDs and typically developing children with sensory intolerance should also be evaluated.

Despite these limitations, the findings of this study have important clinical significance. Video modeling is a costeffective strategy that is likely to reduce caregiver stress and may improve testing compliance. Video modeling may have a direct and indirect effect on child compliance. By improving the overall testing experience for the caregiver, the caregiver may feel better equipped to support their child during the hearing assessments. In addition, the child may directly benefit from pre-exposure to the assessment tasks, the assessor, and the testing environment. Future research should include a larger sample size with a larger age range of children. A larger sample size would also allow for the analysis of factors that may influence compliance, such as the number of times the video was viewed and when the viewing occurred. Future studies should also examine specific components of the video model and delivery methods of the video model that are most effective for reducing caregiver stress and improving child compliance, including instructions for parents around practice, number of views, and timing of the video model.

\section{Abbreviations}

ABR

ASD

$\mathrm{DD}$

DPOAE

NUCASLL

SAT auditory brainstem response autism spectrum disorder developmental delay distortion product otoacoustic emissions

Northwestern University Center for Audiology Speech Language and Learning Speech Awareness Threshold
Acknowledgments

We would like to thank Dean Barbara O'Keefe, Dr. Sumit Dhar, Dr. Dana Brazdziunas, Dr. Sarah Bauer, and Denise Eisenhauer for supporting Northwestern University's Developmental Diagnostics Program, without which this research would not have been possible.

\section{References}

1 Al-Namankany A, Petrie A, Ashley P. Video modelling for reducing anxiety related to the use of nasal masks place it for inhalation sedation: a randomised clinical trial. Eur Arch Paediatr Dent 2014;16(01):13-18

2 American Psychiatric Association. Diagnostic and Statistical Manual of Mental Disorders. Arlington, VA: American Psychiatric Publishing; 2013

3 Baker B, Blacher J, Crnic K, Edelbrock C. Behavior problems and parenting stress in families of three-year-old children with and without developmental delays. J Amer Ment Retard 2002;107 (06):433-444

4 Baker BL, McIntyre LL, Blacher J, Crnic K, Edelbrock C, Low C. Preschool children with and without developmental delay: behaviour problems and parenting stress over time. J Intellect Disabil Res 2003;47(4-5):217-230

5 Bellini S, Akullian J. A meta-analysis of video modeling and video self-modeling interventions for children and adolescents with autism spectrum disorders. Exceptional Child 2007;73(03): 264-287

6 Case L, Yun J. Video modeling and Test of Gross Motor Development-3 performance among children with autism spectrum disorder. Eur J Adapt Phys Act 2019;11(02):1-10

7 Charlop-Christy MH, Le L, Freeman KA. A comparison of video modeling with in vivo modeling for teaching children with autism. J Autism Dev Disord 2000;30(06):537-552

8 Cuvo AJ, Godard A, Huckfeldt R, Demattei R. Training children with autism spectrum disorders to be compliant with an oral assessment. Res Autism Spectr Disord 2010;4(04):681-696 
9 Davis R, Stiegler LN. Toward more effective audiological assessment of children with autism spectrum disorders. Semin Hear 2005;26(04):241-252

10 Demopoulos C, Lewine JD. Audiometric profiles in autism spectrum disorders: does subclinical hearing loss impact communication? Autism Res 2016;9(01):107-120

11 Downs D, Schmidt B, Stephens TJ. Auditory behaviors of children and adolescents with pervasive developmental disorders. Semin Hear 2005;26(04):226-240

12 Dworzynski K, Ronald A, Bolton P, Happé F. How different are girls and boys above and below the diagnostic threshold for autism spectrum disorders? J Am Acad Child Adolesc Psychiatry 2012;51 (08):788-797

13 Fitzpatrick EM, Lambert L, Whittingham J, Leblanc E. Examination of characteristics andmanagement of childrenwith hearing loss and autismspectrumdisorders. Int J Audiol 2014;53(09):577-586

14 Gray C, White AL, McAndrew S. My Social Stories Book. London, UK: Jessica Kingsley Publishers; 2002

15 Harris P, Taylor R, Thielke R, Payne J, Gonzalez N, Conde J. Research electronic data capture (REDCap)-a metadata-driven methodology and workflow process for providing translational research informatics support. J Biomed Inf 2009;42(02):377-381

16 Joint Committee on Infant Hearing. Year 2007 position statement: principles and guidelines for early hearing detection and intervention programs. Pediatrics 2007;120(04):898-921
17 Kayfitz AD, Gragg MN, Orr RR. Positive experiences of mothers and fathers of children with autism. J Appl Res Intellect Disabil 2010;23(04):337-343

18 Kwok S, Ho P, Chan A, Gandhi S, Urn D. Ocular defects in children and adolescents with severe mental deficiency. J intellect disabil Res 1996;40(04):330-335

19 Lord C, Rutter M, DiLavore P, Risi S, Gotham K, Bishop S. Autism Diagnostic Observation Schedule, Second Edition. Torrance, CA: Western Psychological Services; 2012

20 Moeller MP. Early intervention and language development in children who are deaf and hard of hearing. Pediatrics 2000;106 (03):e43

21 Padish-Clarin G, Hawkins J. Retrospective analysis of decreasing the use of anesthesia in pediatric audiology: a preliminary study. Am J Audiol 2015;24(04):557-562

22 R Core Team. R: A Language and Environment for Statistical Computing. Vienna, Austria: R Foundation for Statistical Computing; 2017

23 Rosenhall U, Nordin V, Sandström M, Ahlsé G, Gillberg C. Autism and hearing loss. J Autism Dev Disord 1999;29(05):349-357

24 Tharpe AM, Bess FH, Sladen DP, Schissel H, Couch S, Schery T. Auditory characteristics of children with autism. Ear Hear 2006; 27(04):430-441

25 Zimmerman IL, Steiner VG, Pond RE. Preschool Language Scale, Fifth Edition. San Antonio, TX: Psychological Corp; 2011 